\title{
Visualization Tools for Traffic Bottleneck Analysis
}

\author{
Mark L. Franz ${ }^{1 *}$, Michael L. Pack ${ }^{1}$, Drew Lund ${ }^{1}$ and Catherine Plaisant ${ }^{1,2}$ \\ ${ }^{1}$ Center for Advanced Transportation Technology Laboratory \\ ${ }^{2}$ Human-Computer Interaction Lab, University of Maryland, College Park \\ *mfranz1@umd.edu,packml@umd.edu, alund1@umd.edu,plaisant@cs.umd.edu
}

\begin{abstract}
Traffic bottlenecks are a major source of highway user delay. Therefore, identifying bottlenecks and estimating their impacts is critical for making effective and efficient congestion management decisions. While traditional automated traffic recorders (ATR) provide valuable measurements of traffic flow characteristics, their network coverage is inherently limited. However, increased percentages of vehicles and travelers equipped with global positioning systems (GPS) have enabled transportation analysts to collect continuous and wide spread speed and travel time information from probe vehicles, thus allowing for comprehensive monitoring and analysis of the highway network. This paper presents the development and sample application of novel online bottleneck analysis and visualization tool to assist transportation professionals in identifying bottlenecks, and understanding their impacts using innovative data visualizations based on vehicle probe data analysis. The interactive visualizations include 1) maps illustrating the segments impacted by a given bottleneck(s), 2) timelines displaying the evolution of congestion over one or multiple days, as well as incidents occurring during congestion, 3) charts showing the spatial and temporal impacts of a given bottleneck, and 4) tables summarizing the impacts and rankings of each bottleneck. These visualizations can be used to provide both planning and operational congestion management decision support.
\end{abstract}

Keywords: Traffic Congestion, Data Visualization for Decision Support, Traffic Operations

\section{Background and Objectives}

Traffic bottlenecks plague many of the world's urban areas and are a major source of delay, wasted fuel [1], secondary crashes [2], and increased emissions [3]. With limited resources for congestion mitigation, highway agencies must strategically allocate funds to bottlenecks susceptible to improvement by engineering intervention.

State-of-practice is for transportation analysts to run traffic simulation models or to use engineering judgment to allocate limited resources for congestion mitigation. Simulation takes significant time and effort from highly trained experts to calibrate each bottleneck location to ensure realistic representation of real-world conditions. On the other hand, engineering judgment is subject to potential observational biases regarding which bottlenecks are more severe than others.

To address this issue, we developed an intuitive web-based tool for identifying, analyzing, comparing and visualizing traffic bottlenecks using real-time vehicle probe data. This tool is part of an existing University of Maryland online-suite of probe data tools called the Probe Data Analytics (PDA) Suite that is part of the Regional Integrated Transportation Information System (RITIS). The tool allows real time analysis as well as retrospective analysis to identify and rank bottlenecks for a user-defined region and time period. When this data is combined with additional data sources such as event/incident

* Corresponding Author 
records, traffic signal plans, highway work zone information and weather data, transportation analyst can discover the underlying causes of congestion at each bottleneck location. Such information can be used for traffic operations management and to inform long term transportation investment decisions based on real-world data.

The remainder of the paper is organized as follows. Section 2 summarizes existing studies on bottleneck identification and ranking as well as recent studies on traffic data visualization. Section 3 gives a brief introduction to the proposed web-based bottleneck ranking and visualization tool. Section 4 illustrates the application of the tool using a realworld example. The final section highlights the contributions of this work and defines future research paths.

\section{Related Work}

\subsection{Bottleneck Identification and Ranking}

The first step in any highway congestion mitigation study is to identify when and where traffic bottlenecks occur. While there are varying definitions of what constitutes a bottleneck [4-8], we focus on studies that have been conducted to identify, quantify, and visualize traffic bottlenecks. Lund et al. [8] developed a vehicle probe based algorithm to estimate the relative impacts of bottleneck for the purpose of bottleneck ranking. Ban et al., [9] proposed a bottleneck identification method based on binary speed contour maps (BSCM) and a three step method for bottleneck calibration in traffic simulation software. The related study by Hale et al., [10] developed methods for identifying and quantifying traffic bottlenecks using estimated vehicle hours of delay and assessed low cost solutions for congestion mitigation. Their research created congestion and bottleneck identification (CBI) software to identify and rank bottlenecks based on newly developed data visualizations called the annual reliability matrix (ARM). In addition, several new performance metrics were created to assess the intensity, variability, speed drop, throughput, and unavoidable proportions of delay.

Yet another tool for identifying congestion was developed by Wang et al., [11] that used vehicle trajectory data to find congested roadway segments in a complex urban network. Next, the work of Chen at al. [12] built on the work of Zhang and Levinson [13] to identify bottleneck locations, durations, and cause based on data from adjacent loop detectors. The algorithm was able to identify 160 bottlenecks on freeways near San Diego and by provided guidance on identifying high priority bottlenecks by estimating delay. Studies such as Liu et al., [14] and Ferreira et al., [15] utilized trajectory data from taxi traces to discover areas of high congestion. Finally, the work led by Zhang [16, 17] evaluated bottleneck areas under evacuation events.

\subsection{Visualization of Bottlenecks and Congestion}

Building on studies for identifying and quantifying traffic bottlenecks, several studies have been dedicated to developing visualizations of congestions. Tominski et al., [18] developed a three-dimensional display to visualize the temporal component of traffic evolution. Using this method, time dependent vehicle trajectories were plotted about a two-dimensional network map to illustrate the spatio-temporal relationship of vehicular movements and the evolution of congestion. Guo et al., [19] developed visualizations for spatial, temporal, and multi-dimensional views of traffic data at intersections. Wibisono et al., [20] developed roadway performance maps and charts for several highways in the United Kingdom. Finally, several studies by led by G. Andrienko and N. Andrienko [21, 22] developed novel visualizations for identifying congested roadway segments using spatial and temporal abstraction techniques. 


\section{Overview of the Bottleneck Ranking and Visualization Tool}

Despite the contributions presented in the literature section, there is a need to develop a practice ready bottleneck identification and ranking tool that integrates comprehensive bottleneck identification and quantification techniques with modern data visualization methods. To meet this need, we designed, developed and deployed a web-based tool for querying probe data for the purpose of identifying, ranking and visualizing traffic bottlenecks. The tool is comprised of three main components which are described below and are illustrated in section four:

(1) Data query interface,

(2) Bottleneck identification and ranking algorithm, and

(3) Bottleneck visualization interface

The data query interface allows users to select the road segments of interest as well as the analysis period. Users can select roads segments of interest or spatial clusters of roads within a geographic region. Users can also apply filters such as travel direction, road classification (such as interstates, US routes, frontage roads, etc.,) and zip codes. In addition, the tool allows users to save selected segments for future use. Next, the user can choice a data source. The data sources include several vehicle probe data providers including INRIX, TomTom, and HERE. For each data source, the traffic data is reported for a corresponding traffic message channel (TMC) network that varies for each data provider. For visual confirmation of the query, a map of the selected segments is displayed on a map. Figure 1 displays the data query interface design while Figure 2 presents the associated map of queried segments.

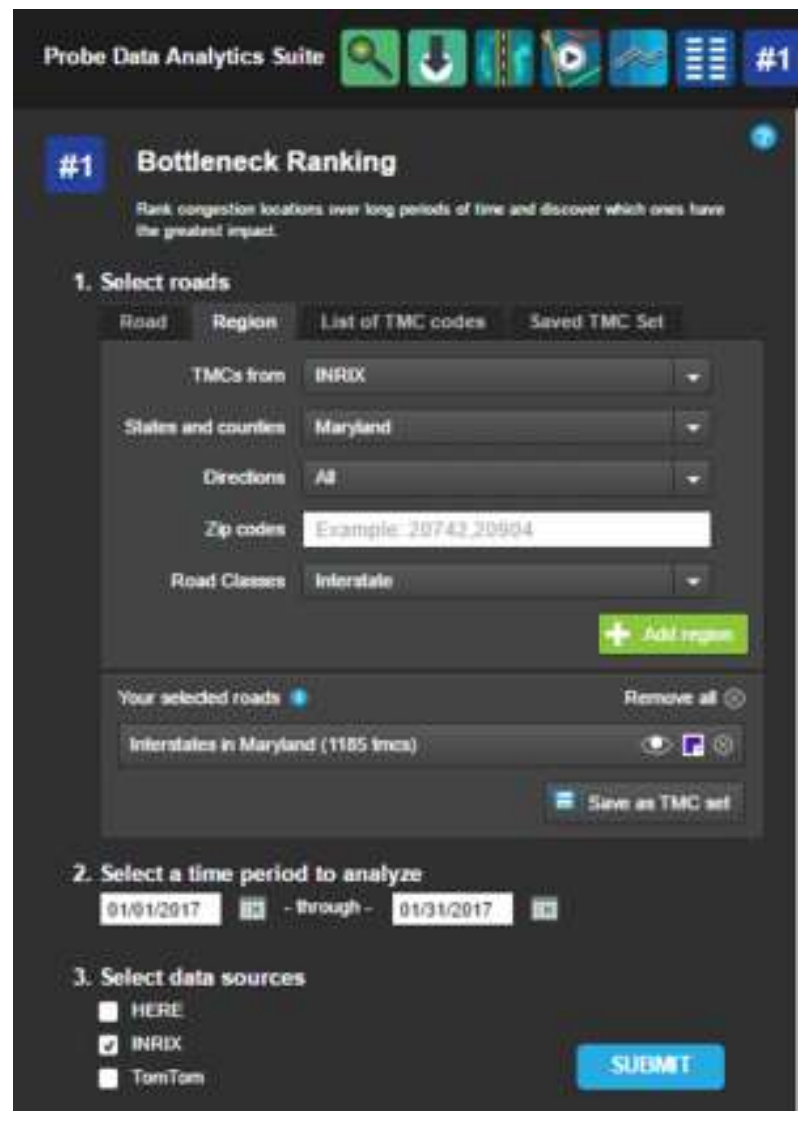

Figure 1. Data Query Interface 


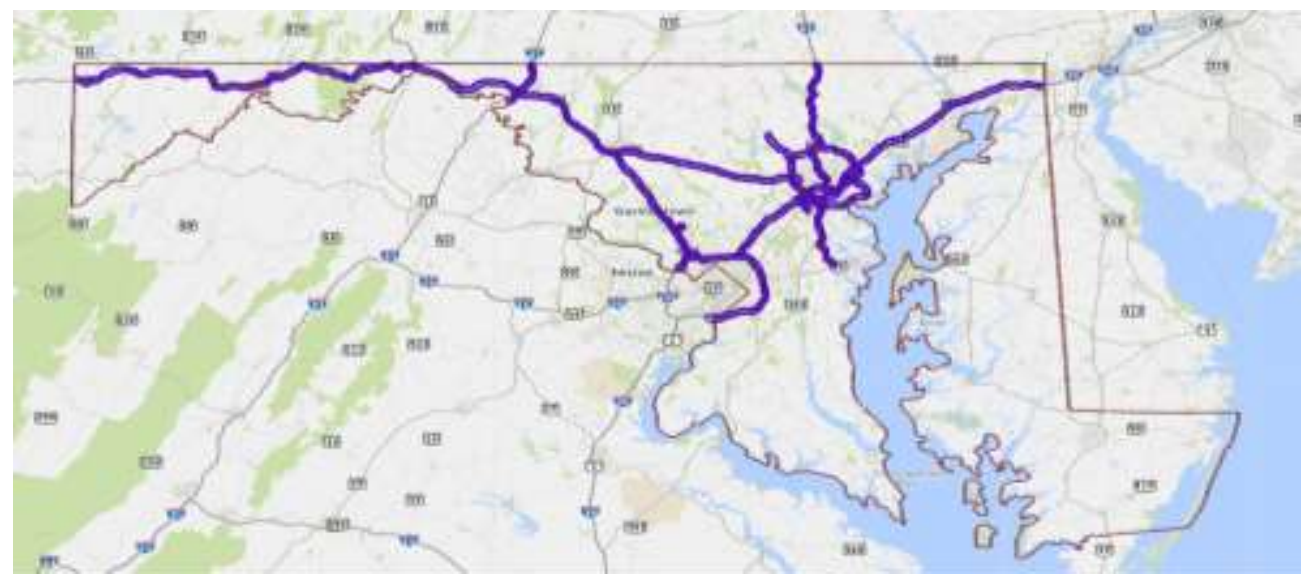

Figure 2. Road Segments Selected using the Query Interface

Next, the queried data is sent to the bottleneck identification and ranking algorithm. While the details of the algorithm are described in details in Lund et al., [8], we include a brief summary. The bottleneck identification process is based on discovering segments with observed speed less than or equal to 60 percent of the reference speed (free-flow speed is often used as the reference speed). Once identified, the algorithm calculates an impact factor based on the results queue length. These procedures are iterated at each data feed update time period (1-3 minutes depending on data provider). To account for the complex evolution of congestion, the algorithm considers the merging and separation of queues from multiple bottlenecks along a heavily congested corridor. To do so, the algorithm defines three critical parameters:

- Occurrence - Congestion, whose head is at a given point on the road at a single point in time

- Element - Congestion, whose head is at a given point on the road, that can change in length over time

- Blob - a collection of spatially and temporally adjacent congestion elements

These three parameters allow for tracking of congestion throughout the entire analysis period to facilitate the accurate estimation of each bottleneck's impact. The general logic of the bottleneck identification and ranking algorithm is conceptualized in Figure 3 while the detailed description of the algorithm can be found in Lund et al., [8].

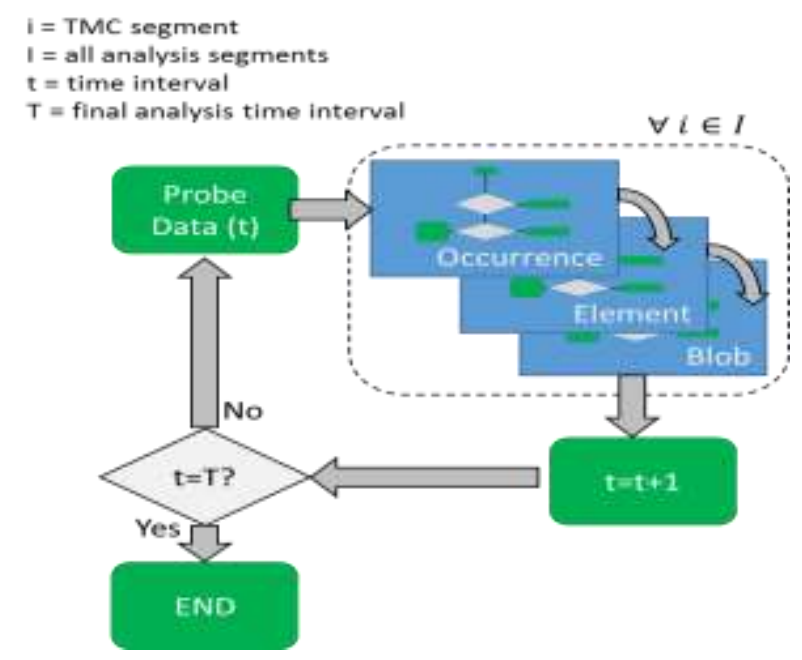

Figure 3. Bottleneck Identification and Ranking Algorithm Process 
Finally, the results of the bottleneck identification and ranking algorithm are passed to the visualization component of the tool. A bottleneck table provides a ranked list of bottlenecks. Analysists can select a bottleneck and review its characteristics using a bottleneck map, a spiral graph, two types of timelines, or an element chart. A screenshot of the visualization component within the PDA Suite is presented in Figure 4. Each component will be discussed in detail in the following section on the sample application. Results and all visualizations can be exported for use in reports and presentations.

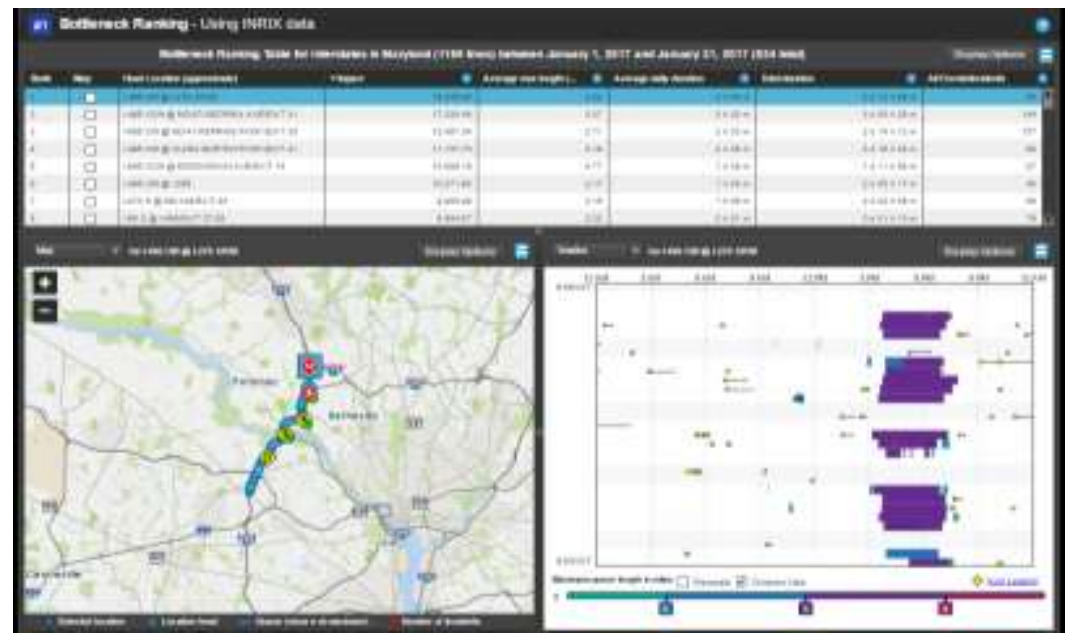

Figure 4. Visualization of Bottlenecks in the PDA Suit

\section{Sample Application of the Bottleneck Ranking and Visualization Tool}

To illustrate the use of the bottleneck ranking and visualization tool, a real-world application is presented. In this application, all interstates in the state of Maryland were analyzed for the month of January 2017 using data from INRIX (see Figures 1 and 2). The remainder of this section will describe and interpret the visualization outputs from the tool.

Upon submitting the bottleneck ranking query, the data is processed in the identification and ranking algorithm and the result is sent to the visualization component. Here, users can inspect the exhaustive list of bottlenecks that occurred under the query parameters. Within the bottleneck ranking table (Figure 5), several metrics are provided to summarize the severity, frequency, duration, number of reported events (such as crashes, roadwork, broken down vehicles, etc.,) and overall impact of each bottleneck.

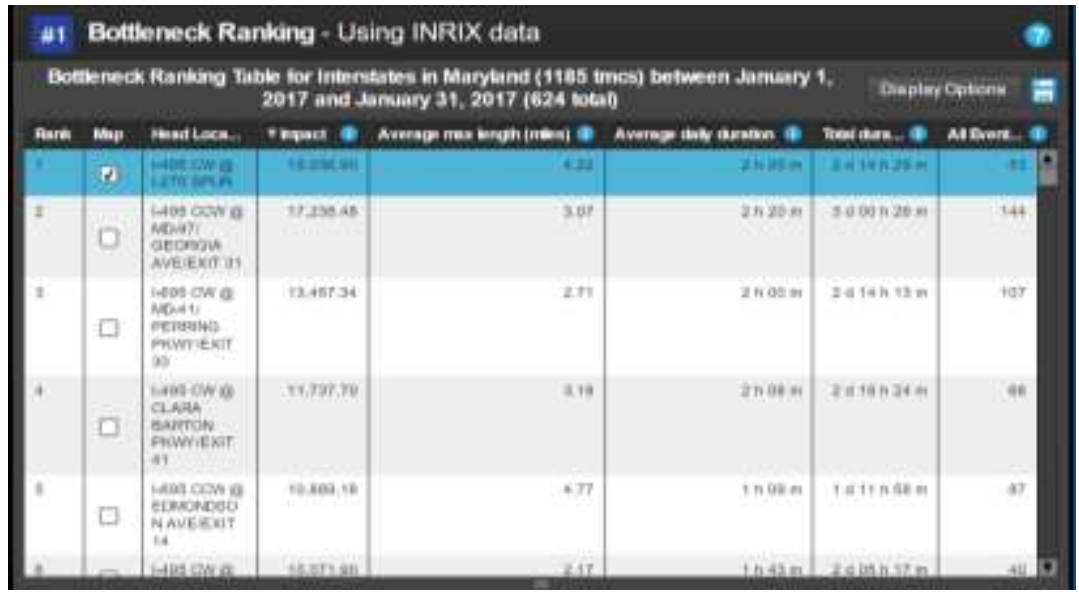

Figure 5. Bottleneck Ranking Table 
As shown in Figure 5, this query analyzed 1,185 TMCs with 624 locations experiencing bottleneck conditions, all of which are presented in the bottleneck ranking table. By default the table is sorted by impact, but other metrics can be used to reorder the table. The top ranked interstate bottleneck in Maryland for January of 2017 was on the Inner Loop (clockwise direction) of I-495 at the spur of the I-270 interchange. At this location, the average maximum queue length was 4.2 miles, with an average daily duration of 2.0 hours. As a result, the total duration of this bottleneck was 2 days, 14 hours and 29 minutes while experiencing 53 reported events. To better illustrate all the features of the interface we use the bottleneck at the Inner Loop of I-495 at the spur of the I-270 interchange as an example to illustrate the additional bottleneck visualizations including the bottleneck map, timeline, time spiral, and elements graph.

Figure 6 shows the bottleneck map. On the map, several metrics are displayed. First, the blue circle with " 1 " represents the location of the head of the top ranked bottleneck. While it is possible to plot multiple bottlenecks, this function is not discussed here. The red diamond with "53" indicates the total number of events (e.g. incidents) that occurred while the bottleneck was active, during the entire analysis period. Yellow and red diamonds are placed at the locations of the aforementioned events. Diamonds with green plus signs represent a cluster of events that can be revealed with a mouse click and displayed in a window showing the list of all events within a given area (Figure 6). Red diamonds indicate events that involved injuries while yellow diamonds represent events with no injuries. Orange diamonds are used to represent maintenance operations, such as work zones. Users can see additional details on each event by clicking on the event of interest. This action opens another tool of the PDA Suite that displays a detailed incident response timeline. Finally, the map shows the upstream TMC segments that were included in the bottleneck at any time during the analysis period. As each cluster of congested segments (i.e. element) adds another layer on the map, road segments become more opaque. Segments closest to the head become the most opaque as they are more frequently affected by congestion at the selected location.

Similar to the events icons, users can learn more about each segment within the queue by hovering the mouse on the segment of interest. Here, a summary of the number of days that the target segment was affected is shown in a pop-out text box (Figure 6).

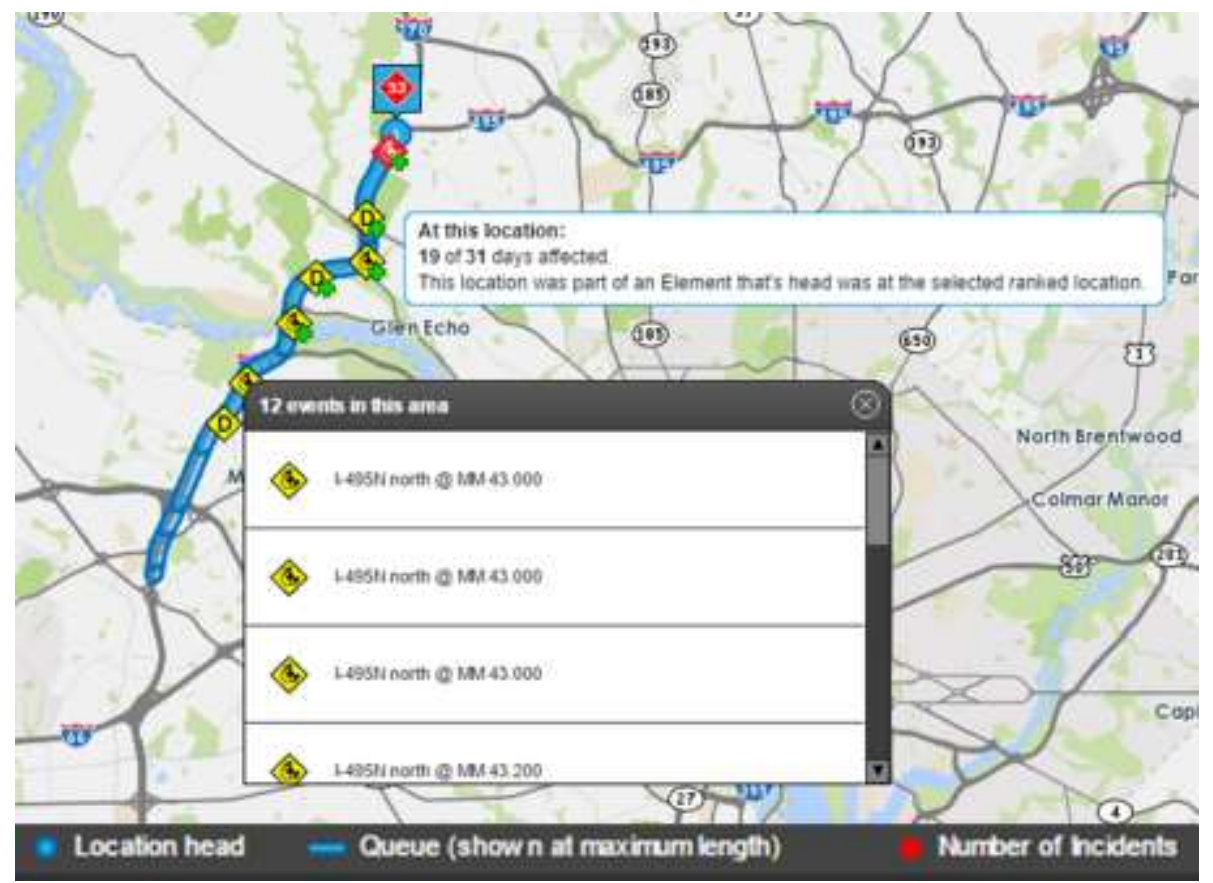

Figure 6. Bottleneck Map 
The next visualizations are the bottleneck timeline and time spiral. These visualizations for Inner Loop of I-495 at the spur of the I-270 interchange is shown in Figure 7 and 8, respectively. In the timeline (Figure 7), the hour of the day is shown on the $\mathrm{x}$-axis while the $y$ axis shows all the days in the analysis period., the queue length for each bottleneck activation period is shown as a colored bar during the associated date and time period. The various colors of the bar represent binned queue lengths (in miles) as described on the legend. Similar to the bottleneck map, the timeline shows events as various colored diamonds. Here, the duration of each event is shown by a line protruding from the diamond. To gain further details on any of the items within the display, including events and bottlenecks, the user can hover the mouse over the item of interest.

Both the timeline and time spiral can be used to identify the general nature of congestion at the bottleneck location, and how events may have affected the bottleneck. For example, Figure 7 shows that an incident (with injuries involved) occurred on Friday January 13, 2017 at 10:52AM and was cleared at 11:14AM (a duration of 22 minutes). While this event occurred during off-peak hours, a bottleneck formed and grew to over two miles long. We can also see a pattern of recurrent congestion during the PM peak hours of 4-7PM for every weekday. Users can hover over any of the bottleneck queues to gather more information such as bottleneck duration, maximum queue length, and impact factor. The bottleneck shown in Figure 8 had a duration of 3 hours and 34 minutes, a maximum queue length of 7.7 miles, and an impact factor of 1,381. While this location experienced 53 events, few occurred during the peak period. An overwhelming majority of the bottleneck activations for this location were caused by re-current congestion, not by events.

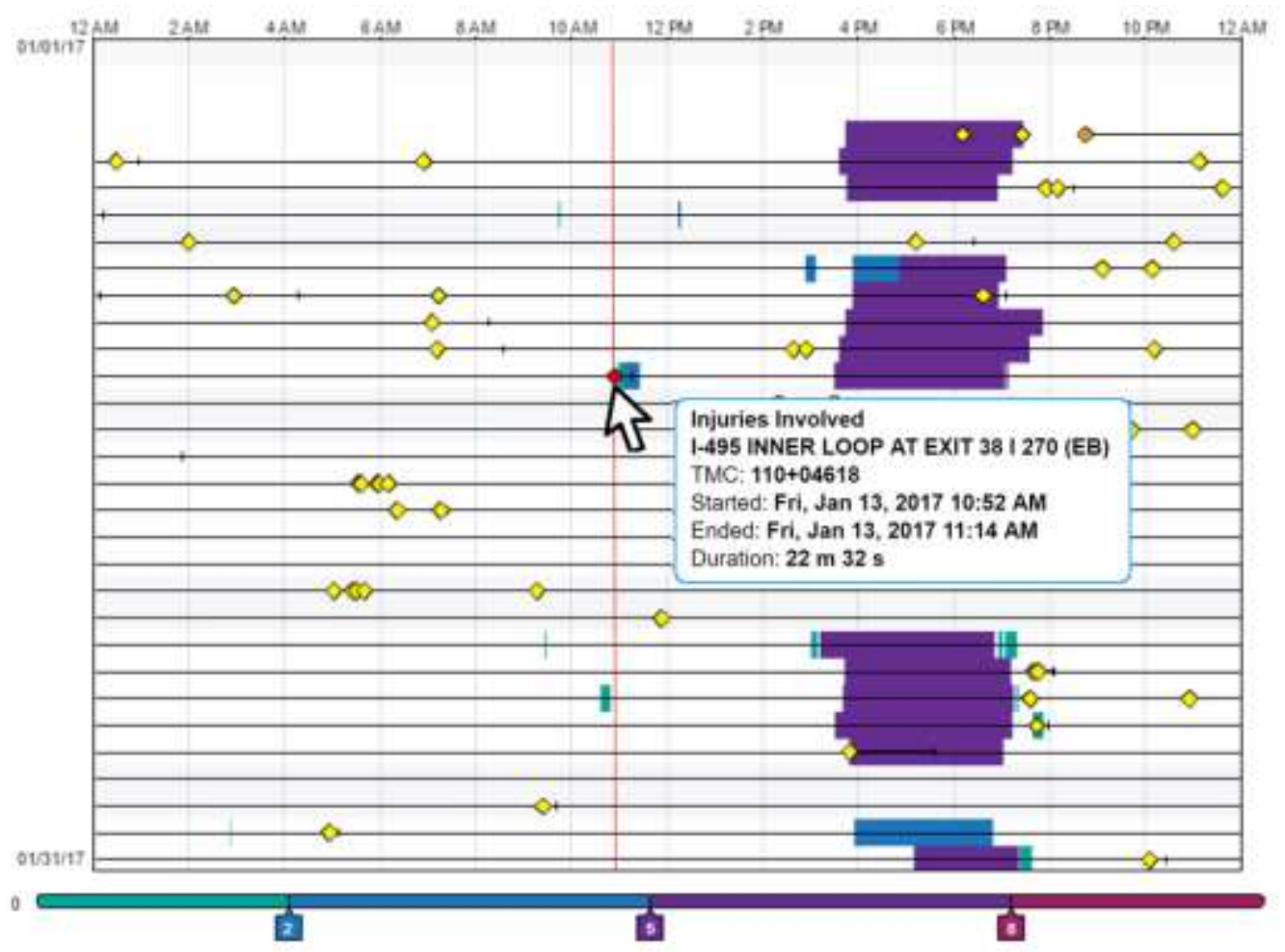

Figure 7. Bottleneck Timeline

The time spiral (Figure 8) is similar to the time line but displays events and bottlenecks on a circular ring based plot. Each concentric ring represents a day and the radii depict an hour of the day. Here, January 1, 2017 is at the center ring and February 1, 2017 is at the outer ring. 


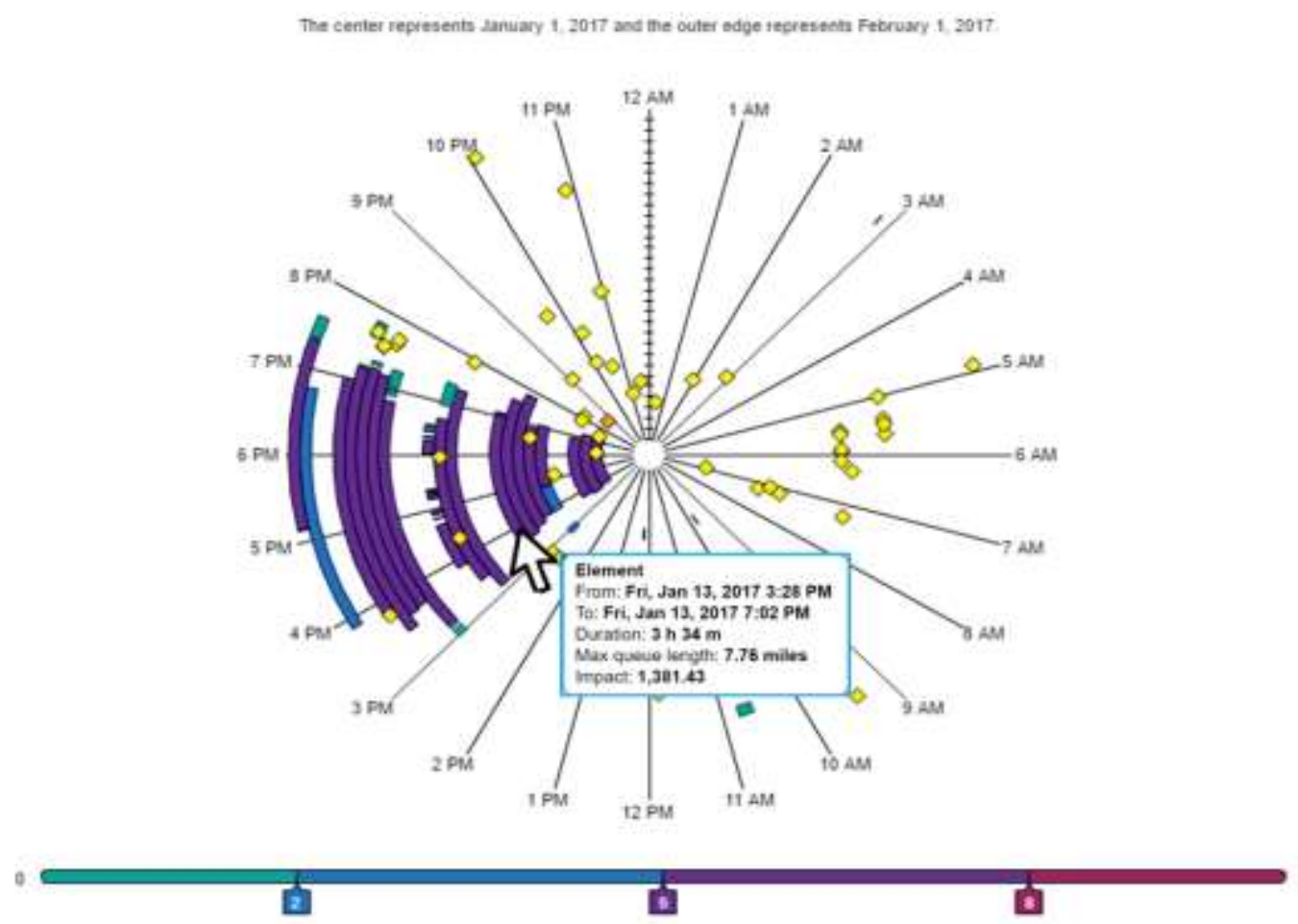

Figure 8. Time Spiral

The fourth and final bottleneck visualization is the elements graph displayed in Figure 9, which was designed and revised in response to users' feedback and suggestions. This comprehensive visualization allows users to see congestion patterns at multiple spatial and temporal levels derived from the work of Shneiderman et al., [23], Bach et al., [24], and Andrienko and Andrienko [25]. The elements graph uses a graphic traffic flow representation of the queued segments associated within the bottleneck (i.e., elements) on the $y$-axis and the time of day on the x-axis. The displayed data is aggregated over all days in the analysis period. The y-axis also presents the independent rank of any bottleneck location that falls within the queue (orange circles) of the bottleneck being analyzed (blue circle). The more opaque orange bands indicate more days of congestion. In addition, the graphic at the top of the element graph displays the average queue length for each hour of the day while a graphic on the right of the element graph shows total hours of congestion for each segment within the queue. Similar to the previous visualizations, more details can be obtained by hovering the mouse over the location and time of interest. As shown in Figure 9, the selected segment approaching the Cabin John Parkway/Exit 40 was congested 17 out of 31 days at 4:54PM. It is worth noting that the segment with the highest number of congested hours occurred in the middle of the queue. One would generally expect the head of the bottleneck to have the most hours of congestion. This observation can be explained by the proximity of the fourth ranked interstate bottleneck in the state of Maryland for January 2017, located approximately three miles upstream of top ranked bottleneck at the Inner Loop of I-495 at the spur of the I-270 interchange. Here, the total number of congested hours is increased by the periods in which this segment was an independent bottleneck. Traditional bottleneck visualizations would likely have not captured this complex congestion pattern. 


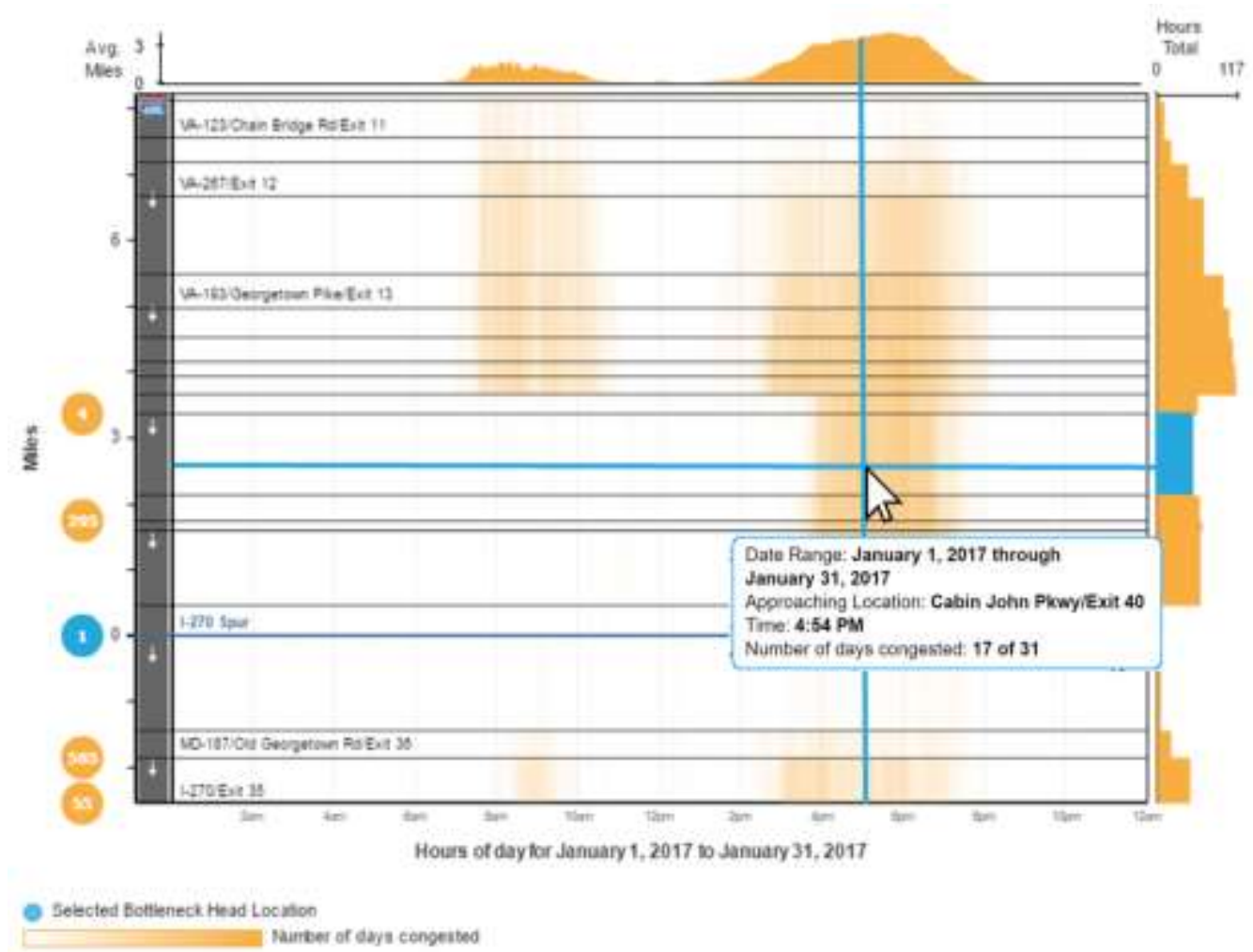

Figure 9. Elements Graph

\section{Conclusions and Future Work}

This paper has presented a novel web-based traffic bottleneck analysis interface that marries rigorous traffic engineering principles with state-of-the-art data visualization. The end product provides transportation analyst and decision makers with a powerful tool to identify, quantify, rank, and visualize traffic bottlenecks. To illustrate the application of the bottleneck analysis and ranking tool, a real-world example was presented. The resulting visualizations were used to discover nature of congestion at a target bottleneck including the contributions of non-recurrent congestion such as queues caused by incidents, and the contribution of re-current congestion to the overall bottleneck impact.

While the bottleneck ranking algorithm described here (and in [8]) is a recent update as well as the element graph, the basic bottleneck ranking tool has been in use by traffic professionals for several years. For example, it is used regularly by the Maryland Department of Transportation - State Highway Administration to create its Annual Maryland Mobility Report [6]. Figure 10 shows monthly usage data from January 2016 to March of 2017. On any given month, over 4,000 traffic professionals utilized this tool to analyze and visualize bottlenecks. The new visualizations have received generally positive feedback from end users. 


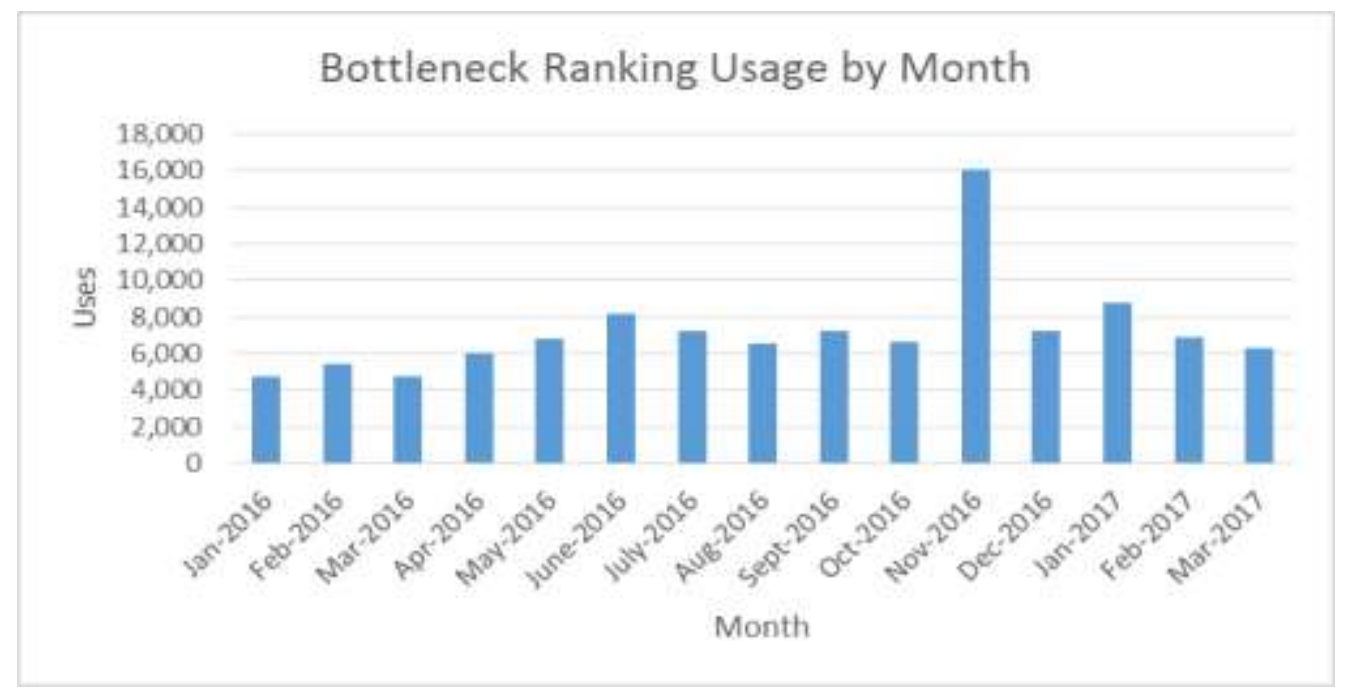

Figure 10. Bottleneck Tool Usage by Month

Future research paths include improvements to the bottleneck ranking algorithm to include the cost of delay. In addition, trajectory data is now becoming available and opening new frontiers by offering trip specific data with regular waypoints. Such data can be used to enhance current bottleneck identification, analysis and visualization techniques by offering anonymous individual trip level travel patterns. This information has the potential to assist transportation analysts and decision makers in understanding traveler behaviors that contribute to congestion such as origin-destination patterns and departure times.

\section{References}

[1] D. Schrank, B. Eisele, T. Lomax and J. Bak, "2015 Urban Mobility Scorecard”, Texas A\&M Transportation Institute, College Station, TX, (2015).

[2] V. Shah, G. Hatcher, E. Greer, J. Fraser, M. Franz and K. Sadabadi, "Guidance on Quantifying Benefits of TIM Strategies”, National Cooperative Highway Research Program Report 03-108. Washington, D.C. (In Press).

[3] M. Grote, I. Williams, J. Preston and S. Kemp, "Including congestion effects in urban road traffic CO 2 emissions modelling: Do Local Government Authorities have the right options?", Transportation Research. Part D, no. 43, (2016), pp. 95-106.

[4] R. Bertini, "Toward the Systematic Diagnosis of Freeway Bottleneck Activation", Proceedings of the IEEE 6th International Conference on Intelligent Transportation Systems, (2003).

[5] X. Ma, H. Yu, Y. Wang and Y. Wang, "Large-Scale Transportation Network Congestion Evolution Prediction Using Deep Learning Theory", PLoS ONE, vol. 10, no. 3, (2015).

[6] S. Mahapatra, M. Wolniak, E. Beckett and K. Sadabadi, 2015 Maryland State Highway Mobility Report., Maryland Department of Transportation, (2016).

[7] J. H. Banks, "New Approach to Bottleneck Capacity Analysis", California PATH Final Report UCBITS-PRR-2006-13, (2006).

[8] A. Lund, M. Pack, C. Plaisant and M. Franz, "Algorithms for Identifying and Ranking Bottlenecks Using Probe Data", Transportation Research Board 96 $6^{\text {th }}$ Annual Meeting, Washington, D.C., (2016).

[9] X. J. Ban, L. Chu and H. Benouar, "Bottleneck Identification and Calibration for Corridor Management Planning", Transportation Research Record: Journal of the Transportation Research Board, No. 1999, Transportation Research Board of the National Academies, Washington, D.C. (2007), pp. 40-53.

[10] D. Hale, R. Jagannathan, M. Xyntarakis, P. Su, X. Jiang, J. Ma, Jia Hu and C. Krause, "Traffic Bottlenecks: Identification and Solutions", Federal Highway Administration Report No. FHWA-HRT16-064, (2016).

[11] Z. Wang, M. Lu, X. Yuan, J. Zhang and H. Van De Wetering, "Visual Traffic Jam Analysis Based on Trajectory Data", IEEE Transactions on Visualization and Computer Graphics, vol. 19, no. 12, (2013) December, pp. 2159-2168.

[12] C. Chen, A. Skabardonis and P. Varaiya, "Systematic Identification of Freeway Bottlenecks", Transportation Research Record: Journal of the Transportation Research Board, No. 1867, TRB, National Research Council, Washington, D.C., (2004), pp. 46-52. 
[13] L. Zhang and D. Levinson, "Some Properties of Flows at Freeway Bottlenecks", Transportation Research Record: Journal of the Transportation Research Board, No. 1883, TRB, National Research Council, Washington, D.C., (2004), pp. 122-131.

[14] S. Liu, J. Pu, Q. Luo, H. Qu, L. M. Ni and R. Krishnan, "VAIT: A Visual Analytics System for Metropolitan Transportation", IEEE Transactions on Intelligent Transportation Systems, vol. 14, no. 4, (2013) December, pp. 1586-1596.

[15] N. Ferreira, J. Poco, H. T. Vo, J. Freire and T. Silva, "Visual Exploration of Big Spatio-Temporal Urban Data: A Study of New York City Taxi Trips", IEEE Transactions On Visualization and Computer Graphics, vol. 19, no. 12, (2013), pp. 2149-2158.

[16] Z. Zhang, S. Parr and B. Wolshon, "Application of a Productivity Function to Assess Network Performance During Mass Evacuations”, International Journal of Transportation, vol. 4, no. 3, (2016), pp. 33-50.

[17] Z. Zhang, K. Spansel, V. Dixit and B. Wolshon, "Performance Characteristics of Megaregion Traffic Networks During Mass Evacuations”, International Journal of Transportation, vol. 2, no. 3, (2014), pp. 53-72.

[18] C. Tominski, H. Schumann, G. Andrienko and N. Andrienko, "Stacking-Based Visualization of Trajectory Attribute Data", IEEE Transactions on Visualization and Computer Graphics, vol. 18, no. 12, (2012) December, pp. 2565-2574.

[19] H. Guo and Z. Wang, "TripVista: Triple Perspective Visual Trajectory Analytics and Its Application on Microscopic Traffic Data at a Road Intersection”, IEEE Pacific Visualisation Symposium, (2011), pp 163-170.

[20] A. Wibisono, W. Jatmiko, H. A. Wisesa, B. Hardjono and P. Mursanto, "Traffic big data prediction and visualization using Fast Incremental Model Trees-Drift Detection (FIMT-DD)”, Knowledge-Based Systems, vol. 93, no. 33, (2016), pp. 33-46.

[21] N. Andrienko, G. Andrienko and S. Rinzivillo, "Leveraging Spatial Abstraction in Traffic Analysis and Forecasting with Visual Analytics", Information Systems, vol. 57, (2016) April, pp. 172-194.

[22] G. Andrienko, N. Andrienko, P. Bak, D. Keim and S. Wrobel, "Visual Analytics of Movement", Springer, New York City, NY., (2013).

[23] B. Shneiderman, C. Plaisant, M. S. Cohen, S. Jacobs, N. Elmqvist and N. Diakopoulos, "Designing the User Interface: Strategies for Effective Human-Computer Interaction”, Pearson, (2016).

[24] B. Bach, P. Dragicevic, D. Archambault, C. Hurter and S. Carpendale, "A Review of Temporal Data Visualizations Based on Space-Time Cube Operations", In EuroVis 2014 - State of the Art Reports, The Eurographics Association, (2014), pp. 1-21.

[25] N. Andrienko and G. Andrienko, "Exploratory Analysis of Spatial and Temporal Data: A Systematic Approach", Springer Science \& Business Media, (2006).

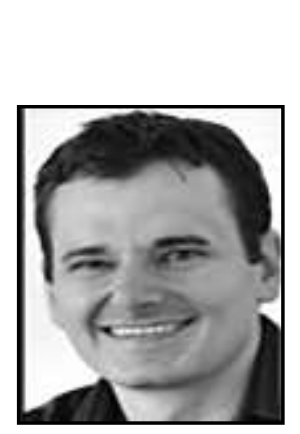

\section{Authors}

Mark Franz earned his BA in Physics and BS in Astronomy from the University of Florida, his MSCE from West Virginia University, and his Ph.D. in Civil and Environmental Engineering from the University of Maryland (UMD) with emphasis on transportation engineering. Currently, Mark is a Transportation Analyst at the Center for Advanced Transportation Technology Laboratory (CATT Lab) at UMD where he is developing and improving online transportation analysis tools and visualizations for public and private sector clients.

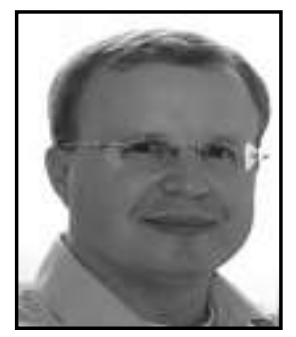

Michael Pack is the director of the Center for Advanced Transportation Technology Laboratory (CATT Lab) at the University of Maryland and the Chair of the Visualization Committee for the National Academies Transportation Research Board. He works to improve transportation operations and make data more readily available, usable, and understandable to diverse communities through visualization and visual analytics. He has previously worked for the University of Virginia's Smart Travel Laboratory and the Oak Ridge National Laboratory Center for Transportation Analysis. 


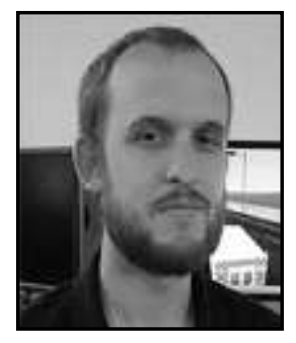

Andrew Lund has 9 years of experience in transportation data management, analytics, performance measures, and traffic operations. He currently serves as a lead developer and senior data architect for the University of Maryland CATT Laboratory where he designs and manages over 40 distinct data storage systems hosting over $200 \mathrm{~TB}$ of probe speed data, traffic detector readings, road incident reports, and other transportation related data. Mr. Lund leads a development team working to implement big-data analytics solutions to support traffic planners and roadway operators. He also provides outreach and support to MPOs, State DOTs, and the Federal Government regarding the use of the NPMRDS and other large probe vehicle data sets, calculating and interpreting the proposed MAP-21 system performance measures, and how to handle data conflation challenges between state, national, and TMC-based reporting networks.

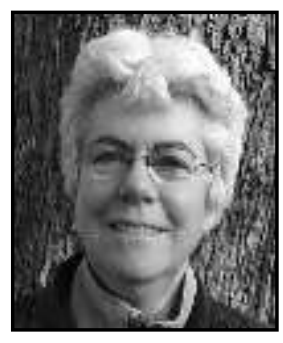

Catherine Plaisant is a Senior Research Scientist at the University of Maryland Institute for Advanced Computer Studies and Associate Director of Research of the Human-Computer Interaction Lab. She is also member of the Center for Advanced Transportation Technology Laboratory. Catherine Plaisant earned her PhD in Industrial Engineering at the Université Pierre et Marie Curie - Paris VI, France. In 2015 she was elected to the ACM SIGCHI Academy for her contributions to the field of humancomputer interaction, medical informatics and information visualization. 